\title{
Gender-related endocrinological dysfunction and mental disorders
}

\author{
Maria Luisa Figueira and Sílvia Ouakinin
}

Department of Psychiatry, Faculty of Medicine, Hospital Santa Maria, University of Lisbon, Lisbon, Portugal

Correspondence to Professor Dr Maria Luisa Figueira Department of Psychiatry, Faculty of Medicine

Hospital Santa Maria, University of Lisbon, Av. Prof. Egas Moniz, P-1649-035 Lisbon, Portugal Fax: +351217990612;

e-mail: ml.figueira@hsm.min-saude.pt

Current Opinion in Psychiatry 2010, 23:369-372

\section{Purpose of review}

The association between endocrine and neuropsychiatric disorders was established long ago, with solid evidence. According to a multidimensional model of mental disorders, one can conceptualize sex and gender-related endocrinological dysfunctions as a cluster of risk factors included in the biological determinants of those disorders. Recent findings

Gender and sexual dimorphism in brain functions and pathways may have a main impact and synergistic effects on health differences in both men and women. To explain these differences, hormonal reactivity to stress, sex hormones and gene-environment interactions are among the most researched mechanisms.

\section{Summary}

In this paper, we review updated data on sex and gender differences in stress reactivity, concerning the hypothalamic-pituitary-adrenal axis, endocrinological dysfunction and vulnerability to major psychiatric disorders, in a stress-diathesis approach. Mainly schizophrenia and affective disorders are discussed, according to recent investigation, in terms of early determinants of stress reactivity, the interplay of genetic expression and gender role, their responsibility in biological modulation and their hypothetical contribution to explain gender differences in prevalence and clinical aspects of mental disorders.

\section{Keywords}

endocrine dysfunction, gender, mental disorders, sex, stress

Curr Opin Psychiatry 23:369-372

(C) 2010 Wolters Kluwer Health | Lippincott Williams \& Wilkins $0951-7367$

\section{Introduction}

The association between endocrine and neuropsychiatric disorders was established long ago, with solid evidence. In a multidimensional model of mental disorders, one can conceptualize sex and gender-related endocrinological dysfunctions as a cluster of risk factors included in the biological determinants of those disorders [1-3].

With regard to mental health, sex and gender are related but distinct concepts that include a number of risk factors of a different nature. Recent research tends to emphasize the interaction between those risk factors and their impact on the development of mental disorders $[4,5]$.

\section{Neuroendocrinological dysfunction and mental disorders}

Different hormonal profiles and biological events, in each sex, can explain some of the differences in presentation of mental disorders and gender-related resilience or vulnerability. Stress reactivity is one of the most important aspects of vulnerability and it can be understood accord- ing to its biological nature but also according to specific aspects in development and maintenance of gender roles $\left[6,7,8^{\bullet \bullet}, 9\right]$.

\section{Stress and the hypothalamic-pituitary- adrenal axis}

The response of the hypothalamic-pituitary-adrenal (HPA) axis to stressful stimuli is essential to maintain physiological stability in the human body.

Physiological reactivity in response to acute stressors is different in males and females. Kajantie and Phillips [10] reviewed sex differences in the response of the HPA axis and the autonomic nervous system to acute psychosocial stress and they concluded that, despite some differences determined by the stressor itself, there are important differences in both sexes with a clear effect of hormonal status according to menstrual cycle, menopause or pregnancy. Adult women between puberty and menopause have a reduced HPA and autonomic reactivity compared with men within the same age group. Before puberty and after menopause, sex differences are small or 
nonexistent, but, in pregnancy, the stress responses are attenuated, perhaps, according to the authors, as a result of evolutionary pressure, to protect the fetus from the negative effects of maternal stress reactivity [10]. According to different authors, investigation showed that corticosteroid-binding globulin, estrogens and arginine vasopressin (AVP) seem involved in HPA axis regulation and exhibit different patterns in both sexes, in animal models of stress reactivity and in humans $[11,12]$.

Concerning the nature of stimuli, Uhart et al. [6] demonstrated that, in response to different stressors, men showed a stronger HPA axis response to psychological stressors and women showed greater hormonal reactivity in response to pharmacological stimulation with naloxone.

It is well documented that fetal exposure to sex steroids is responsible for sexual dimorphism, in both brain structure and function. There is growing evidence that maternal stress increases corticosteroid levels in the fetal brain, decreases testosterone levels in men and alters brain catecholamine activity in women [13].

The relationship between stress and sex, in animal models of stress pathway dysregulation, related to the experience of prenatal stress or early-life stressful events, highlights periods of developmental vulnerability resulting in sex differences for stress sensitivity or neuropsychiatry disease vulnerability. In fact, studies in humans and rodents showed evidence that increased stress responsiveness or inability to respond to stress and maintain homeostatic balance can induce negative brain effects $\left[14^{\bullet \bullet}\right]$. The impact of the stress insult is dependent on the developmental timing, which becomes evident for neurodevelopmental disorders such as schizophrenia. A recent investigation [15] revealed an association between maternal stress during the first trimester and an increased risk of schizophrenia in males.

Goel and Bale $\left[14^{\bullet \bullet}\right]$ proposed that stress system dysregulation and consequent disease vulnerability might be explained through epigenetic changes related to hormonal modulation of stress circuits as well as gene expression at critical developmental points.

Several investigations reviewed by Heim et al. $\left[16^{\bullet}, 17^{\bullet \bullet}\right]$ showed that, in humans, childhood trauma is associated with sensitization of the neuroendocrine stress response, glucocorticoid resistance, increased central corticotropinreleasing factor activity, immune activation and reduced hippocampal volume. For these authors, the risk of developing depression in response to stress may be secondary to neuroendocrine changes associated with early-life stress, eventually due to the failure of a connected neural circuitry involved in emotional, neuroendocrine and autonomic control, as well as other risk factors, such as female sex and genetic dispositions, further increasing vulnerability for depression. In several psychiatric and medical disorders, frequently related to depression and aggravated by stress, similar associations are described $\left[16^{\bullet}, 17^{\bullet \bullet}\right]$.

According to recent research, the role of a functional polymorphism in the promoter region of the serotonin transporter gene (5HTTLPR) is controversial, with inconsistent results, but corticotrophin-releasing hormone receptor 1 (CRHR1) gene and sex interact in moderating effects of childhood trauma on adult depression [17 ${ }^{\bullet \bullet}$ ].

\section{Mental disorders: sex and gender matters}

Gender differences in prevalence, clinical manifestations and evolution in mental disorders are well known. Depression, anxiety and affective disorders, schizophrenia, substance abuse or eating disorders have distinctive patterns in men and women, probably because of genetic and biological determinants but also because of psychosocial and cultural factors $[2,18,19]$.

Reported gender-based differences in mental health may arise from several levels: biomedical (genetic, hormonal, anatomical and physiological), psychosocial (personality, coping and symptom reporting), epidemiological (population-based risk factors) or from a more global viewpoint [2]. Psychosocial stressors, the burden of stressful lifeevents, stress reactivity, coping strategies and their impact on health are different in men and women $[10,20]$.

In order to clarify the relationship of gender and sexual orientation with treatment received for substance use or mental disorders, Grella et al. [21] conducted a populationbased survey, applying a behavioral model for vulnerable populations to 2074 participants aged 18-64 years, in California. They concluded that sexual orientation and gender had the main effect on receiving treatment in the last year, after controlling for the presence of mental disorders and other predisposing factors [21].

\section{Affective disorders}

Differences in the rates of affective disorders between women and men may be related to gender differences in gonadal steroid levels that regulate brain monoamines implicated in mood regulation (serotonin, norepinephrine, dopamine, acetylcholine, $\gamma$-aminobutyric acid and glutamate). The association of a higher risk for affective instability in premenstrual or perimenopausal women has been attributed to fluctuations in $17-\beta$-estradiol. Even if the effects of estrogens on mood appear to be complex and not fully understood, the hypothesis for an increased risk for depression during some reproductive stages has been explained by the cycling levels of gonadal 
steroids on neurotransmitter systems possibly interacting with genetic vulnerability and life stress [22]. One of the challenging issues concerns the identification of the complex underlying neuronal mechanisms responsible for hormone-stress interaction $\left[2^{\circ} 3^{\circ}\right.$. Animal research has indicated that both gender and reproductive steroids regulate basal HPA axis and stimulate its function. In women, the regulatory effects of changes in reproductive steroids or menstrual cycle phase in the HPA axis are less well studied and understood. The findings of a luteal phase increase in HPA axis activity are contradictory, with several kinds of stressors; although some studies identified increased stimulated cortisol, others failed to find a luteal phase increase in HPA axis activity [24 $4^{\circ}$. Women with premenstrual dysphoric disorder (PMS), a combination of both affective symptoms with low stress adaptation during the luteal phase, are a growing field for research in determining the pathophysiological role of reproductive steroids in HPA axis regulation. Studies of HPA axis abnormalities characteristic of major depression conducted with PMS patients are inconclusive to date. Evidence from animal studies suggests that females are relatively resistant to the neurobiological effects of acute and chronic stress as opposed to males, who seem to have a greater HPA axis response to stress, despite the sex differences for the prevalence of affective and anxiety disorders [1]. Other risk factors such as childhood trauma associated with genetic disposition can interfere with components of stress response and increase vulnerability for depression in women [ $\left[16^{\bullet}\right]$. There is a need for further research clarifying the role of reproductive hormones for stress-related biological changes and emotional behavior in order to understand the nature of gender-related affective disorders.

\section{Schizophrenia}

Research on causal aspects of schizophrenia has been focused at predisposing genes and environmental risk factors. In humans as well as in animal models, schizophrenia has been linked with maternal gestational stress and it is generally accepted that human vulnerability may be higher during the first trimester of gestation. Hormonal responses following acute psychological stress, namely the HPA axis and sex hormone regulation, their impact on brain development and epigenetic programming during early prenatal stress, are important pathways to explain these facts $\left[15,25^{\circ}, 26\right]$.

Malaspina $\mathrm{et} \mathrm{al.}\left[25^{\circ}\right]$ tried to clarify the role of an acute maternal stress, investigating, in a cohort prospective study, the offspring of women who were pregnant at a time of war. Data from their cohort of 88829 infants displayed a raised incidence of schizophrenia for those in the second month of fetal life, higher in females than in males, suggesting both sex specificity and a relatively narrow window of vulnerability, even after controlling for potential confounders $\left[25^{\circ}\right]$.

Discussing the importance of the interaction between genetic and environmental factors, Oh and Petronis [27 ${ }^{\bullet \bullet}$ ] stated that 'The epigenetic model of schizophrenia can be thought of as a result of a chain of deviant epigenetic events, which begins with a preepimutation (an epigenetic change that takes place during gametogenesis or embryogenesis). A preepimutation increases the risk for schizophrenia, but it is not sufficient to cause the disease. The phenotypic outcome, that is, presence or absence of the disease, is dependent on the overall effect of a series of prenatal and postnatal factors on the preepimutation. The epigenetic pattern is altered over time by the external environmental factors, stochastic events and hormones, further increasing or decreasing the degree of the epigenetic misregulation' $\left[27^{\bullet \bullet}\right]$.

With regard to cognitive functions and disease evolution, the literature addressing sex differences in schizophrenia generally reports male patients as being more impaired than female patients. Bozikas et al. [28] investigated 96 patients with schizophrenia and gender-matched healthy controls to observe whether differences in cognitive performance in healthy men and women are preserved in patients with schizophrenia. Using a battery of neuropsychological tests to assess attention, working memory, abstraction, inhibition, fluency, verbal learning, memory, visuospatial skills and psychomotor speed, it was concluded that the degree of cognitive impairment was the same for male and female patients with schizophrenia and sex differences found among the patients were similar to those found in healthy controls. Therefore, the authors considered that differential decrements in basic cognition were not accountable for the favorable course of schizophrenia in women compared with men [28].

Several investigations support the fact that there are gender differences in age of onset, severity of symptoms and long-term functioning in schizophrenia, with different patterns of prodromal symptoms beginning before the disorder onset [29,30]. As Lewine [31] has already indicated, several aspects of schizophrenia, such as the onset, may be best pursued from a biological (sex difference) perspective, whereas a sociocultural perspective, including gender differences, may be better to understand different aspects, such as neuropsychological functioning.

\section{Conclusion}

Gender and sexual dimorphism in brain functions and pathways may have the main impact and synergistic effects on health differences in men and women. 
Research in sex-linked biological determinants for psychiatric disorders has progressed during recent years yielding new insights in etiopathogenic mechanisms. Nevertheless, gender-related determinants appear to have been less clarified mainly because of methodological issues. Interactions between biological, psychological and sociocultural factors are complex and current paradigms have, as yet, insufficient explanatory power.

\section{References and recommended reading}

Papers of particular interest, published within the annual period of review, have been highlighted as:

- of special interest

-. of outstanding interest

Additional references related to this topic can also be found in the Current World Literature section in this issue (p. 401).

1 Altemus M. Sex differences in depression and anxiety disorders: potential biological determinants. Horm Behav 2006; 50:534-538.

2 Afifi M. Gender differences in mental health. Singapore Med J 2007; 48:385391.

3 McLaughlin KA, Conron KJ, Koenen KC, Gilman SE. Childhood adversity, adult stressful life events, and risk of past-year psychiatric disorder: a test of the stress sensitization hypothesis in a population-based sample of adults. Psychol Med 2009; 17:1-12.

4 Vigoda S, Stewart DE. Emergent research in the cause of mental illness in women across the lifespan. Curr Opin Psychiatry 2009; 22:396-400.

5 Dedovic K, Wadiwalla M, Engert V, Pruessner JC. The role of sex and gender socialization in stress reactivity. Dev Psychol 2009; 45:45-55.

6 Uhart M, Chong RY, Oswald L, et al. Gender differences in hypothalamicpituitary-adrenal (HPA) axis reactivity. Psychoneuroendocrinology 2006; $31: 642-652$

7 Jezova D, Hlavacova N. Endocrine factors in stress and psychiatric disorders: focus on anxiety and salivary steroids. Ann N Y Acad Sci 2008; 1148:495503.

8 Darnall BD, Suarez EC. Sex and gender in psychoneuroimmunology research: .• past, present and future. Brain Behav Immun 2009; 23:595-604.

This study provides an overview on the literature of sex and gender in brain structure and function, sex steroids, gender role identification, HPA axis function, genetics, immunology and cytokine response.

9 Goldstein JM, Jerram M, Abbs B, et al. Sex differences in stress response circuitry activation dependent on female hormonal cycle. J Neurosci 2010; 30:431-438.

10 Kajantie E, Phillips DIW. The effects of sex and hormonal status on the physiological response to acute psychosocial stress. Psychoneuroendocrinology 2006; 31:151-178.

11 Makara GB, Domokos A, Mergl Z, et al. Gender-specific regulation of the hypothalamo-pituitary-adrenal axis and the role of vasopressin during the neonatal period. Ann N Y Acad Sci 2008; 1148:439-445.

12 Zelena D, Domokos A, Barna I, et al. Control of the hypothalamo-pituitaryadrenal axis in the neonatal period: adrenocorticotropin and corticosterone stress responses dissociate in vasopressin-deficient brattleboro rats. Endocrinology 2008; 49:2576-2583.

13 Weinstock M. Gender differences in the effects of prenatal stress on brain development and behaviour. Neurochem Res 2007; 32:1730-1740.

14 Goel N, Bale TL. Examining the intersection of sex and stress in modeling -• neuropsychiatric disorders. J Neuroendocrinol 2009; 21:415-420.

A review paper concerning the neurobiology of stress-related disease predisposition, as well as the interaction between sex and stress, in animal models of disease.
15 Khashan AS, Abel KM, McNamee R, et al. Higher risk of offspring schizophrenia following antenatal maternal exposure to severe adverse life events. (Reprinted) Arch Gen Psychiatry 2008; 65:146-152.

16 Heim C, Newport DJ, Mletzko T, et al. The link between childhood trauma and

- depression: insights from HPA axis studies in humans. Psychoneuroendocrinology 2008; 33:693-710.

This is a review paper on the relationship of HPA studies on humans, stress response to childhood trauma and depression.

17 Heim C, Bradley B, Mletzko TC, et al. Effect of childhood trauma on adult

- depression and neuroendocrine function: sex-specific moderation by $\mathrm{CRH}$ receptor 1 gene. Front Behav Neurosci 2009; 3:41.

In this investigation, the CRHR1 gene-moderating effect, in the relationship between childhood trauma and depression is analyzed, in men and women, pointing to a gene-sex interaction.

18 Schimmele CM, Wu Z, Penning MJ. Gender and remission of mental illness. Can J Public Health 2009; 100:353-356.

19 Blehar MC. Women's mental health research: the emergence of a biomedical field. Annu Rev Clin Psychol 2006; 2:135-160.

20 de Visser L, van der Knaap LJ, van de Loo AJ, et al. Trait anxiety affects decision-making differently in healthy men and women: towards genderspecific endophenotypes of anxiety. Neuropsychologia 2010. [Epub ahead of print]

21 Grella CE, Greenwell L, Mays VM, Cochran SD. Influence of gender, sexual orientation, and need on treatment utilization for substance use and mental disorders: findings from the California Quality of Life Survey. BMC Psychiatry 2009; 9:52.

22 Caspi A, Sugden K, Moffitt TE, et al. Influence of life stress on depression: moderation by a polymorphism in the 5 -HTT gene. Science 2003; 301:386389.

23 Newhouse PA, Dumas J, Hancur-Bucci C, et al. Estrogen administration

- negatively alters mood following monoaminergic depletion and psychosocial stress in postmenopausal women. Neuropsychopharmacology 2008; 33:1514-1527.

This study examined whether $17-\beta$-estradiol (E2) administration can modify the mood effects of experimental psychosocial stress following acute monoamine depletion in postmenopausal women.

24 Rubinow DR, Schmidt PJ, Meltzer-Brody S, Harsh VL. Hypoyhalamic-pitui-

- tary-gonadal axis in women. In: Rubin RT, Pfaff DW, editors. Hormone/ behavior relations of clinical importance. Endocrine systems interacting with brain and behaviour. Amsterdam: Elsevier; 2009. pp. 85-118.

A review chapter on the role of gonadal steroids in female behavior.

25 Malaspina D, Corcoran C, Kleinhaus KR, et al. Acute maternal stress in - pregnancy and schizophrenia in offspring: a cohort prospective study. BMC Psychiatry 2008; 8:71.

This study describes an investigation into the role of acute maternal stress, in a cohort prospective study, in the offspring of women who were pregnant during a period of war.

26 Mueller BR, Bale TL. Sex-specific programming of offspring emotionality after stress early in pregnancy. J Neurosci 2008; 28:9055-9065.

27 Oh G, Petronis A. Environmental studies of schizophrenia through the prism of •• epigenetics. Schizophr Bull 2008; 34:1122-1129.

An important paper discussing the interaction between genetic and environmental factors, from an epigenetic perspective and addressing epigenetic regulation in schizophrenia.

28 Bozikas VP, Kosmidis MH, Peltekis A, et al. Sex differences in neuropsychological functioning among schizophrenia patients. Aust N Z J Psychiatry 2010 44:333-341.

29 Choi JS, Chon MW, Kang DH, et al. Gender difference in the prodromal symptoms of first-episode schizophrenia. J Korean Med Sci 2009; 24:10831088.

30 Willhite RK, Niendam TA, Bearden CE, et al. Gender differences in symptoms, functioning and social support in patients at ultra-high risk for developing a psychotic disorder. Schizophr Res 2008; 104:237-245.

31 Lewine R. At issue: sex and gender in schizophrenia. Schizophr Bull 2004; 30:755-762. 